\title{
Cognitive impairment as a function of sleep quality and gender
}

Akhil $\mathrm{R}^{1^{*}}$, Nair BP ${ }^{2}$

*Corresponding author:

${ }^{1}$ Mr. Akhil. R, MSc, Psychologist, Psychologist, Department of Psychology, Kariavattom Campus, University of Kerala, Thiruvananthapuram.

Email: akhilrajeev9099@gmail.com ORCID

${ }^{2}$ Associate Professor, Department of Psychology, Kariavattom Campus, University of Kerala, Thiruvananthapuram, Kerala, India

Information about the article:

Received: Jan. 17, 2019

Accepted: Feb. 20, 2019

Published online: Dec. 27, 2019

Publisher

Nepal Health Research Society, Bahundhara -6, Gokarnesowor Municipality, Kathmandu, Nepal eISSN 2382-5545, ISSN 2676-1343 (Print)

(c) The Author(s). 2019

Content licensing: CC BY 4.0

\begin{abstract}
Background

The study aims at finding whether there is any significant difference between sub-groups classified on the basis of sleep quality (good sleep quality and poor sleep quality) and gender in the performance of various cognitive functioning tests like Visual N Back Test (N Back 1and N Back 2 test) for working memory, Triad test for divided attention, Digit Vigilance Test for sustained attention and Reaction time test (simple reaction time and choice reaction time).
\end{abstract}

\section{Materials and methods}

The sample consisted of 30 participants, both males $(\mathrm{N}=13)$ and females $(\mathrm{N}=17)$ in the age range between 18 to 30 years, randomly drawn from Thiruvananthapuram and Kollam districts of Kerala. The participants of the study are screened and categorized into two groups of 15 members each on the basis of the scores obtained in the Pittsburgh Sleep Quality Index (PSQI). t-test and two-way ANOVA were performed to test the significance of the hypotheses.

\section{Results}

The results showed that the participants with poor sleep quality significantly differed from those with good sleep quality and committed more number of errors in the triad test of divided attention and took more time and committed more errors in the completion of the digit vigilance test of sustained attention.

\section{Conclusion}

A gender advantage favoring females was seen on the test of working memory, test for sustained attention and the test for choice reaction time. An interaction between sleep quality and gender was noticed only on the test of divided attention. It was seen that males with poor sleep quality are more impaired in divided attention tasks than females with poor sleep quality.

\section{Keywords}

Divided attention, PSQI, reaction, time, sleep quality, sustained attention, working memory 